\title{
Author Index Vol. 28, 1996
}

Abiko,T. 160 Abraham, E.C. 230 Akeo,K. 8 Akiba,M. 289 Alió,J.L. 64 Armstrong, D. 184 Augustin, A.J. 71 Azuma, M. 289 Azzi,A. 171

Babizhayev, M.A. 365 Balas,D. 130 Bannigan, J. 255 Barrett, R. 50 Beck,D. 193 Beckers, H.J.M. 36 Beigi, B. 255 Benelli,U. 176 Bettelheim, F.A. 219 Böhnke,M. 171 Booth, R. 44 Boscoboinik, D. 171 Boulton, M.E. 296 Bours, J. 365 Brandt, C.R. 125 Bron,A.M. 331

Budak,K. 80 Buzney, S.M. 108

Çagil,N. 351 Caldemeyer, K. 193 Carpineto, P. 312 Chen,E. 209 Chen,H.-B. 29 Chen,M.-S. 137 Cheng, F.Y. 57 Cheng, H.-M. 57 Chetoni,P. 176 Ciancaglini, M. 312 Clayton, R.M. 237 Cohen, RJ. 19 Comoli, A.M. 224 Costagliola, C. 312 Cuthbert,J. 237

De Angelis, M.S. 224 DeWing,M.D. 117 Dogan,H. 351 Doi,M. 201 Donnelly, C.A. 237

Doughty, M.J. 141 Dybdahl,A. 319

Ekinciler, Ö.F. 351 Engen,D. 193 Erkúiç,K. 351 Eryümaz, T. 80 Eustace, P. 255 Evans, D. 193

Fagerholm, P. 361 Feke,G.T. 108,343 Fernandes, R. 275 Foreman, D.M. 296 Fredj-

Reygrobellet, D. 130 Fujii,Y. 29 Fulcher,T. 255

Gallenga, P.E. 312 Garcher,C. 331 Garrett, M. 50 Giordano, M. 224 Glonek,T. 44 Goger,D.G. 108,343 Green, K. 230 Greiner, J.V. 44 Gribbestad, I.S. 319 Gündüz,K. 80

Haas,A.L. 171 Hangai,M. 248 Harris, A. 193 Hayasaka,S. 147,260 Hayashi,H. 356 Hazlett,L. 50 Hiraki,S. 147,260 Hiramitsu, T. 8 Hirata,H. 147 Honda, Y. 248 Hou,P.-K. 137 Hristova,D. 130

Huang, J.-K. 137 Hung,P.-T. 137 Hurwitz, M.Y. 19 Hurwitz, R.L. 19

Ikoma, N. 153 Ishiguro, I. 336 Ishikawa, M. 117 Ishiko,S. 160 Ismail, M.M. 64

Javouhey, A. 331 Johnson, L.V. 117 Jones, S. 296

Kado,M. 160 Kamiya, S. 284 Kanda,T. 8 Karasawa, Y. 8 Kato,K. 270 Kellerer, A.M. 265

Kin,L.L.-K. 137 Kiritoshi,A. 201 Kishida,K. 201 Kitaya,N. 160 Klooster, J. 36 Koch,F.H.J. 71

Kommonen, B. 19 Konno, S. 108 Konoeda,Y. 284 Korb,D.R. 44 Koseki,T. 117 Kretschmann, U. 303 KumpfmüUer, H. 265 Kurpakus, M. 50 Kylmä,T. 19

Lamers, W.P.M.A. 36 Leahy, CD. 44 Lepri, A. 176 Lewden, O. 331 Li,W. 2 Liu,X. 2 Lobefalo, L. 312

McKechnie, N.M. 296 McMeel,J.W. 108,343 Majima,Y. 336 Manna, C. 93 Martin, B. 193

Masinick, S. 50 Mastropasqua, L. 312 Meller,D. 71 Mezger, B. 50 Michael, R. 209 Midelfart,A. 319 Mirza,G.E. 351 Mojon,D.S. 171 Momigliano Richiardi, P. 224 Morales, C. 331 Mori,F. 160 Mota, M.C. 275 Munz,K. 99 Murayama, T. 153 Mutani, R. 224

Nardi, M. 176 Niimi, K. 336 Niwa,T. 336

Numata-Watanabe, K. 147 Nunziata, G. 93

Ogasawara, H. 108,343 Ogasawara, M. 289 Ogawa,T. 284 Oguni,M. 270 Ohnishi,Y. 165 Ohno, S. 29 Ohta,Y. 336 Okada,A.A. 284 Okada,Y. 165 Okisaka,S. 8 Oliveira, C.R. 275 Ooshima, A. 165 Oshima,K. 356 Otani,H. 270 Ou,B. 29

Ozaki,H. 356 Özkan, M. 80 
Paulin,L. 19 Penn,J.S. 19 Pereira,P.C. 275 Perini, G. 176 Philipson, B. 361 Phillips, C.I. 237 Pierscionek, B.K. 88 Prescott, R.J. 237 Pumfery,A.M. 125

Qian,W. 237

Raad, S. de 99 Ramalho,J.S. 275 Remé,C.E. 99 Rinaldi,E. 93 Rochette,L. 331 Romano, N. 93

Roos, H. 265 Ruiz Moreno, J.M. 64 Rüther,K. 303

Saettone, M.F. 176 Saika,S. 165 Saito,K. 160 Sakuragi, S. 117 Sasabe,T. 201 Schultz,G 361

Sebastiani, F. 224 Senegas-Balas, F. 130 Setogawa, T. 270 Setten, G.-B. van 361 Shih, Y.-F. 137

Shinohara, H. 270 Simonelli,F. 93 Söderberg, P.G. 209 Suwa,Y. 201 Suzutani,T. 289 Swamy-

Mruthinti, S. 230

Swartz, D. 193 Szczesny, P.J. 99

Takahashi,M. 160 Takahashi,N. 153 Tanaka, S.-i. 165 Tanaka,T. 284 Ten Tusscher, M.P.M. 36

Terada, N. 29 Tonosaki, A. 117 Tsukahara, S. 29

Uçakhan,Ö. 80 Ueda,T. 184 Usui,M. 284 Usui,T. 303 Utikal,K.J. 365

Voto,O. 93

Vrensen, GF.J.M. 1,36

Want, J.J.L. van der 36 Weale, R.A. 88 Williams, G. 296

Xiong,H. 57 Xiong,J. 57

Yamabayashi, S. 29 Yamaguchi, H. 284 Yamakawa, N. 284 Yamanaka, O. 165 Yamasaki, T. 336 Yanoff,M. 2 Yoshida,A. 108, 160,343 Yoshida,I. 289 Yoshimura, N. 248 Yuasa,T. 201

Zhang, X.Y. 260 Zigler, J.S., Jr. 219 Zrenner, E. 303

376

Author Index Vol. 28, 1996 